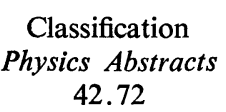

\title{
SPECTRE DE LA RAIE D DU Rb DANS LE CAS D'UNE LAMPE SPHÉRIQUE
}

\author{
I. HIRANO (*) \\ National Research Laboratory of Metrology \\ 1-10-4, Kaga, Itabashi-ku, Tokyo, Japan
}

(Reçu le 31 janvier 1977, révisé le 18 mai 1977, accepté le 31 mai 1977)

\begin{abstract}
Résumé. - Les profils spectraux des raies $D_{1}$ et $D_{2}$ du $R b$ et les distributions radiales des 'cnsités des atomes de $\mathrm{Rb}$ pour une lampe sphérique avec $\mathrm{Rb}-\mathrm{Ar} 267 \mathrm{~Pa}$ à $387 \mathrm{~K}$, sont obtenus théoriquement. Les analyses montrent que le degré d'auto-absorption peut être déduit de la température de la paroi de la lampe, c'est-à-dire, par le nombre des atomes dans l'état fondamental près de la puroi, quand les distributions radiales des densité des atomes excités et ceux dans l'état fondame: tal sont connues. Il semble aussi que les lignes spectrales d'émission et d'absorption sont déplacées relativement l'une par rapport à l'autre même dans la lampe et ce déplacement est estimé être environ 1,2 mk pour la lampe mentionnée ci-dessus. On a supposé que les distributions radiales des densités des atomes excités et ceux dans l'état fondamental augmentent à partir du centre de la lampe vers la paroi de façon quadratique. Le nombre des atomes excités au centre de la lampe est estimé être moins de $1 / 10$ de celui près de la paroi et une zone d'environ $100 \mu \mathrm{m}$ près de la paroi est occupée à peu près exclusivement par des atomes dans l'état fondamental.
\end{abstract}

Abstract. - Spectral profiles of $R b-D_{1}$ and $D_{2}$ lines and radial density distributions for a spherical lamp with $\mathrm{Rb}-\mathrm{Ar} 267 \mathrm{~Pa}$ at $387 \mathrm{~K}$, are obtained theoretically. The analysis reveals the fact that the degree of self-absorption is deduced from the wall temperature of the lamp, that is, from the number of atoms in the ground state near the wall when the radial density distributions of both the excited and ground state atoms are known. It also appears that the emission and the absorption spectral lines are displaced relatively to each other even in the same lamp and this displacement is estimated to be about $1.2 \mathrm{mk}$ for the lamp mentioned above. The radial density distributions of the excited and the ground state atoms are assumed to increase quadratically from the center of the lamp to the wall.

The number of excited state atoms in the center of the lamp is estimated to be below $1 / 10$ of those at the wall and a zone of about $100 \mu \mathrm{m}$ near the wall is occupied almost exclusively by atoms in the ground state.

1. Introduction. - Dans les standards de fréquence à pompage optique de vapeurs alcalines, le profil des raies est intimement lié à l'efficacité du pompage optique et aux phénomènes de glissement de fréquence du standard dûs aux variations d'intensité de la lampe. Il est donc nécessaire d'étudier le profil des raies résonnantes des atomes alcalins. D'autre part, les raies résonnantes sont un sujet important des études spectroscopiques de l'effet d'auto-absorption, de l'évaluation d'élargissement et de déplacement par collision atomique et du potentiel d'interaction entre des atomes, etc...

De très nombreuses études sur l'élargissement et le déplacement des raies spectrales ont déjà été publiées.

(*) Adresse actuelle : Institut d'Electronique Fondamentale, Laboratoire associé au C. N. R. S., Bâtiment 220, Université Paris XI, 91405 Orsay Cedex, France (Boursier du Gouvernement français).
En particulier, on peut citer principalement les articles de Margenau et Watson [1], de Ch'en et Takeo [2] et de Sobélman [3]. Mais ils n'abordent pas suffisamment l'effet d'auto-absorption [4-5] et ne semblent pas considérer le fait qu'il puisse y avoir un déplacement relatif du spectre d'émission et du spectre d'absorption dans la lampe émettrice.

Cet article discute l'influence des effets indiqués ci-dessus en effectuant une analyse théorique sur la raie $\mathrm{D} d u \mathrm{Rb}$, parce qu'on a fait précédemment une expérience [6] avec une lampe sphérique remplie de $\mathbf{R b}$ et de gaz tampon, excitée par un champ électrique de haute fréquence d'environ $100 \mathrm{MHz}$. A titre d'exemple, on prend le cas où $\mathrm{Rb}-\mathrm{Ar} 267 \mathrm{~Pa}, 387 \mathrm{~K}$ avec une puissance continue de $5,9 \mathrm{~W}$. Cet article traite du Rb d'abondance naturelle. Une lampe à isotopes séparés sera étudiée dans l'article suivant.

Par comparaison entre les profils expérimentaux et théoriques, on déduit les distributions radiales des 
atomes excités et des atomes dans l'état fondamental, dans la lampe sphérique. L'effet d'auto-absorption est aussi discuté et on donne une estimation du déplacement relatif des spectres d'émission et d'absorption.

2. Elargissement et déplacement de la raie spectrale. - L'élargissement naturel $\Gamma$ de la raie spectrale, qui provient de la vibration au point zéro du champ électromagnétique, de la raie $D_{1}$ du $R b\left(5^{2} P_{1 / 2}\right.$ $\left.5{ }^{2} \mathrm{~S}_{1 / 2}, 794,7 \mathrm{~nm}\right)$ et de $\mathrm{D}_{2}\left(5^{2} \mathrm{P}_{3 / 2}-5{ }^{2} \mathrm{~S}_{1 / 2}, 790,0 \mathrm{~nm}\right)$ est environ $0,187 \mathrm{mk}(=5,602 \mathrm{MHz})$ et $0,194 \mathrm{mk}$, respectivement. Le déplacement naturel [3] est estimé à environ $10^{-8} \mathrm{mk}$. On exprime le spectre par l'effet Doppler comme une fonction $I_{\mathrm{D}}(v)$. Le déplacement Doppler est $\pm v_{0} v / c$ ( $v_{0}$ est la fréquence centrale non perturbée de la radiation, $v$ la vitesse de l'atome et $c$ la vitesse de la lumière), ce qui produit l'élargissement $\Delta v_{\mathrm{D}}$, c'est-à-dire la largeur Doppler, pour une distribution Maxwellienne des atomes. Le déplacement produit par l'effet Doppler du second ordre [7], est estimé à environ $10^{-5} \mathrm{mk}$ pour la raie $\mathrm{D}$ du $\mathrm{Rb}$ à une température de $387 \mathrm{~K}$.

En outre, la raie spectrale s'élargit et se déplace sous l'effet de la pression. L'effet de pression est dû d'une part aux collisions du rubidium avec le gaz tampon et d'autre part aux collisions avec des atomes de même nature ou aux collisions avec la paroi de la lampe.

Les collisions avec le gaz tampon sont traitées par l'approximation d'impact ou l'approximation statique. L'approximation d'impact est valable dans la région centrale de la raie et pour les basses pressions. La raie spectrale dans l'approximation d'impact est exprimée par $I_{\mathrm{i}}(v)$, qui a une forme de Lorentz et est normalisé par intégration. On estime que la région intermédiaire de la théorie spectrale est éloignée d'environ $190 \mathrm{mk}$ de la fréquence centrale, pour la raie $D$ du $R b$, et de plus, la séparation hyperfine de la raie $\mathrm{D}$ du $\mathrm{Rb}$ reste à l'intérieur de $\pm 160 \mathrm{mk}$, ce qui explique que l'approximation d'impact soit valable.

L'élargissement $\gamma$ et le déplacement $\Delta$ causés par l'interaction dipole-dipole de van der Waals sont donnés par

$$
\begin{aligned}
& \gamma=N v^{3 / 5}\left(\frac{3}{8} \pi C_{6}\right)^{2 / 5} \\
& \Delta \approx-\gamma / 2,8
\end{aligned}
$$

où $v=\sqrt{2 k T / m}$ est la vitesse moyenne, et $N$ le nombre des atomes du gaz tampon dans le volume unitaire.

La valeur de $C_{6}$ est estimée par la force dispersive de London, l'énergie dispersive de Buckingham ou le potentiel Lennard-Jones $[12,6]$, etc..., mais pour l'énergie dispersive de Buckingham,

$$
C_{6}=\frac{3}{2} a_{0}^{1 / 2} \cdot e^{2} \cdot \frac{\alpha_{1} \alpha_{2}}{\left(\alpha_{1} / N_{1}\right)^{1 / 2}+\left(\alpha_{2} / N_{2}\right)^{1 / 2}}
$$

où $a_{0}$ est le rayon de l'orbite primaire de Bohr, $\alpha_{1}$ et $\alpha_{2}$ sont les constantes de polarisation des atomes qui interagissent, et $N_{1}$ et $N_{2}$ sont les nombres d'électrons pour chaque atome.

Pour Rb-Ar, $C_{6} \approx 1,96 \times 10^{-57}$ erg. $\mathrm{cm}^{6}$.

L'élargissement résonnant produit par la collision entre des atomes de $\mathrm{Rb}$, est donné par

$$
\gamma_{R} \approx \frac{3 e^{2}}{m v_{0}} f_{J J_{0}} \sqrt{\frac{2 J+1}{2 J_{0}+1}} N_{J_{0}}
$$

où $e$ est la charge élémentaire, $m$ la masse de l'électron, $f_{J_{0}}$ la force d'oscillateur de la transition $J \rightarrow J_{0}$, et $N_{J_{0}}$ représente le nombre des atomes au niveau $J_{0}$. L'élargissement résonnant ne s'accompagne pas de déplacement. Pour la raie $\mathrm{Rb}-\mathrm{D}_{1}, J=\frac{1}{2}, J_{0}=\frac{1}{2}$, $f_{J J_{0}}=\frac{1}{3}$, et $\gamma_{\mathrm{R}} \approx 2,24 \times 10^{-14} N_{J_{0}} \mathrm{mk}$. Pour la raie $\mathrm{Rb}-\mathrm{D}_{2}, J=\frac{3}{2}, J_{0}=\frac{1}{2}, f_{J J_{0}}=\frac{2}{3}$, et $\gamma_{\mathrm{R}} \approx 4,40 \times$ $10^{-14} N_{J_{0}} \mathrm{mk}$.

$N_{J_{0}}$ est obtenu par la formule de Nernst [8-9], qui est bien vérifiée autour de $400 \mathrm{~K}$ pour le $\mathrm{Rb}$ ou bien par une autre formule [10]. Mais, plusieurs auteurs donnent des valeurs proportionnelles différentes en ce qui concerne l'éq. (2). On utilisera donc ici la formule de Nernst.

La figure 1 montre l'élargissement et le déplacement par collision et l'élargissement résonnant en fonction de la température.

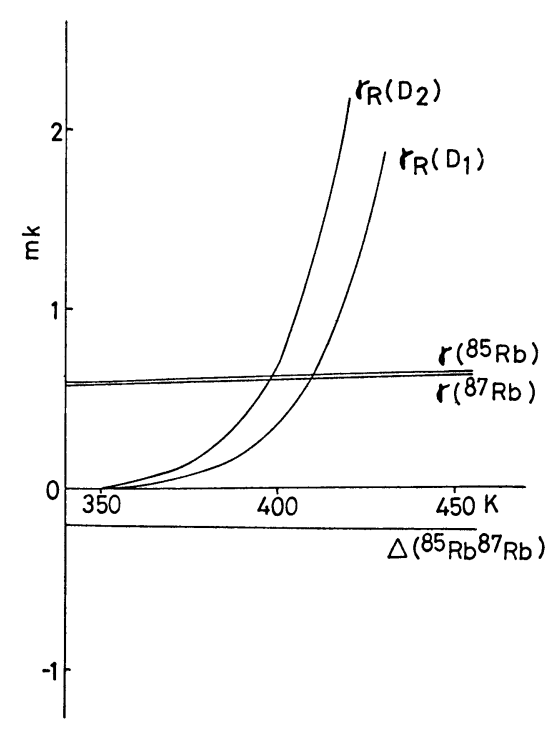

Fig. 1. - Comparaison entre l'élargissement par choc $\gamma$, le déplacement $\Delta$ et l'élargissement résonnant $\gamma_{\mathrm{R}}$. L'ordonnée est en mk et l'abscisse en température Kelvin.

[Comparison between the collision broadening $\gamma$, the shift $\Delta$ and the resonance broadening $\gamma_{\mathrm{R}}$. The ordinate is in mk and the abcissa is in degrees Kelvin.]

L'élargissement par collision avec la paroi de la lampe [11] est exprimé par $\Delta v_{w}$. La lampe utilisée dans cette expérience est de forme sphérique dont le diamètre est $10 \mathrm{~mm}$ environ, ce qui donne pour $\mathrm{Rb}$, $\Delta v_{\mathrm{w}} \approx 10^{-4} \mathrm{mk}$. De plus, le déplacement par collision avec la paroi est plus petit que l'élargissement. 
Dans la lampe, un champ électrique sera provoqué par des ions produits par la décharge. Le profil spectral par l'effet Stark n'est pas exprimé actuellement par une fonction simple. On le négligera ici. On montre ci-dessous que cette approximation est valable dans ce travail.

3. Profils spectraux. - 3.1 Combinaison de L'efFet DOPPLER AVEC L'EFFET DES COLLISIONS. - L'effet Doppler du second ordre et l'effet des collisions avec la paroi sont plus petits que les autres effets, et par conséquent, sont négligeables ainsi que le déplacement naturel. En outre, on peut supposer que l'approximation d'impact soit suffisante pour la raie Rb-D.

Puisque l'élargissement naturel, celui par collision avec le gaz tampon, celui résonnant peuvent être considérés indépendants statistiquement, on peut les additionner pour obtenir l'élargissement résultant.

En conséquence, en écrivant $\gamma_{\mathrm{f}}=\Gamma+\gamma+\gamma_{\mathrm{R}}$, le profil spectral est donné, par

$$
\begin{aligned}
I(v) & =\int I_{\mathrm{i}}\left(v^{\prime}\right) I_{\mathrm{D}}\left(v-v^{\prime}\right) \mathrm{d} v^{\prime} \\
& =\frac{\gamma_{\mathrm{f}}}{2 \pi \sqrt{\pi} \Delta v_{\mathrm{D}}} \int \frac{\exp \left[-\left(\frac{v-v^{\prime}}{\Delta v_{\mathrm{D}}}\right)^{2}\right]}{\left(v^{\prime}-v_{0}-\Delta\right)^{2}+\left(\gamma_{\mathrm{f}} / 2\right)^{2}} \mathrm{~d} v^{\prime} .
\end{aligned}
$$

Cette équation exprime le profil spectral d'une seule composante hyperfine. Dans le cas où toutes les composantes hyperfines sont observées simultanément on obtient :

$$
\begin{aligned}
I(v) & =\sum_{i} I(i) \frac{\gamma_{\mathrm{f}}(i)}{2 \pi \sqrt{\pi} \Delta v_{\mathrm{D}}(i)} \times \\
& \times \int_{-\infty}^{\infty} \frac{\exp \left[-\left(\frac{v-v^{\prime}}{\Delta v_{\mathrm{D}}(i)}\right)^{2}\right]}{\left[v^{\prime}-v_{0}(i)-\Delta(i)\right]^{2}+\left[\frac{\gamma_{\mathrm{f}}(i)^{2}}{2}\right]} \mathrm{d} v^{\prime}
\end{aligned}
$$

où $I(i), \gamma_{\mathrm{f}}(i), \Delta v_{\mathrm{D}}(i), v_{0}(i)$ et $\Delta(i)$ sont l'intensité, l'élargissement par collision, l'élargissement Doppler, la fréquence centrale et le déplacement de la $i$-ième composante, respectivement. Dans ce cas,

$$
\gamma_{\mathrm{f}}(i)=\Gamma(i)+\gamma(i)+\gamma_{\mathbf{R}}(i),
$$

où $\Gamma(i), \gamma(i)$ et $\gamma_{\mathrm{R}}(i)$ représentent l'élargissement naturel, celui par collision avec le gaz tampon et celui résonnant de la $i$-ième composante, respectivement.

Pour la raie $\mathrm{D}_{1}$ et $\mathrm{D}_{2}$ du $\mathrm{Rb}, i=1 \sim 8$ et $i=1 \sim 12$, respectivement.

3.2 AUto-ABSORPTION, Distribution RADiALE DE L'INTENSITÉ DE LA LUMIÈRE EN DEHORS DE L'AXE. Les coordonnées d'un point d'émission de la lampe sont définies par $h, x$ et $r$, comme indiquées sur la figure 2. $C$ représente une limite discutée ci-dessous, où entre $C$ et $R$, il n'y a presque jamais d'atome excité et cette région se trouve remplie par des atomes dans l'état fondamental.

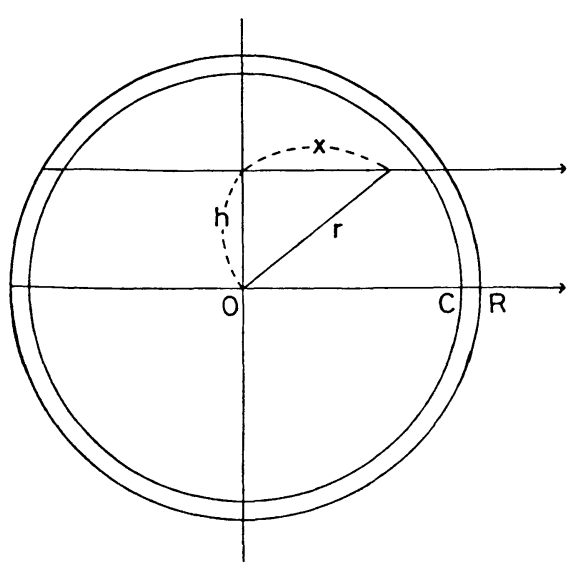

FIG. 2. - Coordonnées dans la lampe sphérique. $O$ : centre de la lampe, $R$ : rayon de la lampe, $C$ : entre rayons $R$ et $C$, il y aura seulement des atomes dans l'état fondamental, $h:$ degré d'hors-axe, $r$ : rayon, $x=\sqrt{r^{2}-h^{2}}$.

[The coordinates for the spherical lamp. $O:$ Center of the lamp, $R$ : Radius of the lamp, $C$ : between radius $R$ and $C$, there will only be atoms in the ground state, $h$ : degree of off-axis, $r$ : radius, $x=\sqrt{r^{2}-h^{2}}$.]

Supposons le spectre d'émission exprimé par $E f_{\mathrm{e}}$ et celui d'absorption par $A f_{\mathrm{a}}$, et la densité en nombre des atomes au point $x$ représentée par $N_{\mathrm{e}}(x)$ et $N_{\mathrm{a}}(x)$ pour les atomes excités et ceux dans l'état fondamental, respectivement. ( $E$ et $A$ sont des constantes, $f_{\mathrm{e}}$ et $f_{\mathrm{a}}$ sont des spectres normalisés : $f_{\mathrm{e}}=f_{\mathrm{e}}(v), f_{\mathrm{a}}=f_{\mathrm{a}}(v)$.) Dans ces conditions, l'intensité de la lumière en dehors de l'axe de la lampe est observée dans la direction paral-

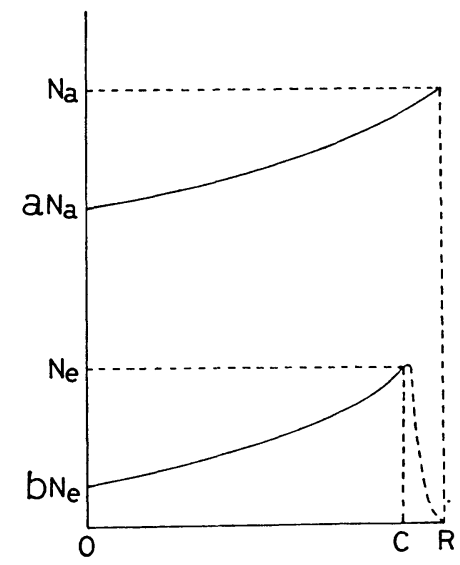

Fig. 3. - Distributions radiales, dans la lampe, des atomes dans l'état excité et de ceux dans l'état fondamental. $O, C$ et $R$ ont les mêmes sens que dans la figure $2 . N_{\mathrm{a}}$ : nombres des atomes dans l'état fondamental près de la paroi. $a N_{\mathrm{a}}$ : nombres des atomes dans l'état fondamental au centre. $N_{\mathrm{e}}$ : nombres des atomes dans l'état excité près de la paroi. $b N_{\mathrm{e}}$ : nombres des atomes dans l'état excité au centre.

[Radial distributions, in the lamp, of the atoms in the excited state and those in the ground state. $\mathrm{O}, \mathrm{C}$ and $R$ have the same meaning in the figure $2 . N_{\mathrm{a}}$ : number of atoms in the ground state near the wall. $a N_{\mathrm{a}}:$ number of atoms in the ground state at the center. $N_{\mathrm{e}}$ : number of atoms in the excited state near the wall. $b N_{\mathrm{e}}$ : number of atoms in the excited state at the center.] 
lèle à cet axe, appelée la distribution radiale de l'intensité est, par suite de l'auto-absorption, donnée par

$$
\begin{aligned}
& I_{\mathrm{a}}(h)=E f_{\mathrm{a}} \int_{-\sqrt{C^{2}-h^{2}}}^{\sqrt{c^{2}-h^{2}}} N_{\mathrm{e}}(x) \times \\
& \times \exp \left[-A f_{\mathrm{a}} \int_{x}^{\sqrt{R^{2}-h^{2}}} N_{\mathrm{a}}\left(x^{\prime}\right) \mathrm{d} x^{\prime}\right] \mathrm{d} x .
\end{aligned}
$$

On peut constater, ici, qu'on ne peut pas déduire la distribution des atomes excités, $N_{\mathrm{e}}(x)$, par l'inversion d'Abél, parce qu'on doit considérer l'effet d'autoabsorption.
Comme il a déjà été rapporté précédemment [6], la distribution des atomes du Rb excités et ceux dans l'état fondamental n'est pas homogène dans la lampe émettrice, leurs nombres, pour les deux, augmentent au fur et à mesure que l'on se rapproche de la paroi. En se référant aux expériences du van Tongeren et al. $[12,13$, 14], on suppose ici qu'ils augmentent d'une manière quadratique vers la paroi, comme le montre la figure 3. Si on suppose que le nombre des atomes excités et celui dans l'état fondamental soit $N_{\mathrm{e}}$ et $N_{\mathrm{a}}$ près de la paroi et $b N_{\mathrm{e}}$ et $a N_{\mathrm{a}}$ au centre de la lampe, respectivement, en mettant $R=1$, on obtient

$$
\begin{aligned}
I_{\mathrm{a}}(h)=E f_{\mathrm{e}} N_{\mathrm{e}} \int_{-\sqrt{c^{2}-h^{2}}}^{\sqrt{c^{2}-h^{2}}}\left\{b+(1-b)\left[\left(\frac{h}{c}\right)^{2}\right.\right. & \left.\left.+\left(\frac{x}{c}\right)^{2}\right]\right\} \times \\
& \times \exp \left\{-A f_{\mathrm{a}} N_{\mathrm{a}} \int_{x}^{\sqrt{1-h^{2}}}\left[a+(1-a)\left(h^{2}+x^{\prime 2}\right)\right] \mathrm{d} x^{\prime}\right\} \mathrm{d} x \\
= & \frac{(1-b) E f_{\mathrm{e}} N_{\mathrm{e}}}{(1-a) c^{2} A f_{\mathrm{a}} N_{\mathrm{a}}}\left\{\exp \left[c_{1}(\alpha-\beta)+c_{2}\left(\alpha^{3}-\beta^{3}\right)\right]-\exp \left[-c_{1}(\alpha+\beta)-c_{2}\left(\alpha^{3}+\beta^{3}\right)\right]\right\} \\
& +\left(b-\frac{a(1-b)}{(1-a) c^{2}}\right) E f_{\mathrm{e}} N_{\mathrm{e}} \int_{-\alpha}^{\alpha} \exp \left[c_{1}(x-\beta)+c_{2}\left(x^{3}-\beta^{3}\right)\right] \mathrm{d} x
\end{aligned}
$$

où

$$
\alpha=\sqrt{c^{2}-h^{2}}, \quad \beta=\sqrt{1-h^{2}}, \quad c_{1}=A f_{\mathrm{a}} N_{\mathrm{a}}\left[1-(1-a) \beta^{2}\right], \quad c_{2}=\frac{1}{3}(1-a) A f_{\mathrm{a}} N_{\mathrm{a}} .
$$

Dans ce cas, la distribution spectrale de la lumière en dehors de l'axe est obtenue, en remplaçant $f_{\mathrm{e}}$ et $f_{\mathrm{a}}$ par $I(v)$ dans l'éq. (3), ainsi qu'il sera discuté ci-dessous.

Or, dans l'éq. (4), si on pose $f_{\mathrm{e}}=f_{\mathrm{a}}, p=A N_{\mathrm{a}}$, $I_{0}=E N_{\mathrm{e}}$ et $p, I_{0}, a$ et $b$ étant des paramètres, on

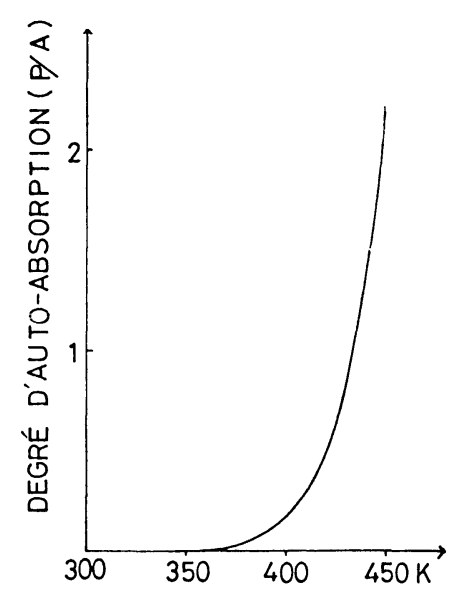

Fig. 4. - Variation avec la température du degré d'autoabsorption de la raie $\mathrm{D}$ du $\mathrm{Rb}$. Ce degré d'auto-absorption est proportionnel aux nombres des atomes dans l'état fondamental près de la paroi de la lampe. L'abscisse est en température Kelvin.

[Variation with the temperature of the degree of self-absorption of the $\mathrm{D}$ line of $\mathrm{Rb}$. This degree of self-absorption is proportional to the number of atoms in the ground state near the wall of the lamp. The abcissa is in degrees Kelvin.] obtient, en fonction de $h$, la distribution de l'intensité de la lumière de la lampe émise parallèlement à l'axe de la lampe. Quand on définit $p$ comme le degré d'autoabsorption [4], on peut obtenir $p$ en fonction du nombre des atomes dans l'état fondamental près de la paroi. La fonction $p / A=N_{\mathrm{a}}$ est représentée dans la figure 4.

4. Effet de l'appareillage. - Les profils mesurés contiennent l'élargissement produit par l'appareillage expérimental, dont il faut tenir compte pour comparer les courbes expérimentales avec les courbes théoriques. Dans les effets de l'appareillage, il y a l'élargissement d'Airy de l'interféromètre de Pérot-Fabry utilisé ; l'élargissement par le diaphragme installé devant le photomultiplicateur détectant les franges d'interférence, et l'élargissement Gaussien produit par l'imperfection du système optique [15].

Dans cette expérience, on a utilisé un interféromètre Pérot-Fabry qui a des couches diélectriques réfléchissantes déposées sur les miroirs plans. Le facteur de réflexion est $95 \%$ pour les raies $D_{1}$ et $D_{2}$ du $R b$. La longueur mécanique de l'étalon interférentiel est $t=10 \mathrm{~mm}$ et le facteur de réflexion $R=0,95$, ce qui donne l'élargissement d'Airy d'environ $8,16 \mathrm{mk}$ pour les raies $D_{1}$ et $D_{2}$ du $R b$.

L'élargissement par un petit diaphragme est donné par [16].

$$
\Delta v_{\mathrm{p}} \approx \frac{s^{2}}{2 f^{2}} v
$$


où $s$ est le rayon du petit diaphragme, $f$ est la distance focale de la lentille qui projette les franges d'interférence sur le diaphragme et $v$ est le nombre d'onde de la lumière utilisée. Le rayon du diaphragme utilisé dans la mesure était $0,5 \mathrm{~mm}$, la distance focale de la lentille convergente $500 \mathrm{~mm}$, et les valeurs de $\Delta v_{\mathrm{p}}, 1,57 \mathrm{mk}$ et $1,60 \mathrm{mk}$ pour la raie $D_{1}$ et $D_{2}$ du $R b$, respectivement. Si le centre des franges interférentielles est déplacé de $0,1 \mathrm{~mm}$ du centre du diaphragme, $\Delta v_{\mathrm{p}}$ est environ
$2,3 \mathrm{mk}$, et au bord du diaphragme il sera environ $6,4 \mathrm{mk}$ La fonction du diaphragme se définit par

$$
f_{\mathrm{p}}(v)=\left\{\begin{array}{l}
1,|v| \leqq \frac{\Delta v_{\mathrm{p}}}{2} \\
0,|v|>\frac{\Delta v_{\mathrm{p}}}{2} .
\end{array}\right.
$$

La convolution de la fonction d'Airy, $A(v)$, avec celle du diaphragme donne :

$$
\begin{aligned}
F_{\mathrm{AP}}(v)=\int_{-\infty}^{\infty} A(v & \left.-v^{\prime}\right) f_{\mathrm{P}}\left(v^{\prime}\right) \mathrm{d} v^{\prime} \\
& =\frac{1}{2 \pi} \cdot \frac{1-R}{1+R}\left\{\tan ^{-1}\left[\frac{1+R}{1-R} \tan 2 \pi\left(v+\frac{\Delta v_{\mathrm{p}}}{2}\right)\right]-\tan ^{-1}\left[\frac{1+R}{1-R} \tan 2 \pi\left(v-\frac{\Delta v_{\mathrm{p}}}{2}\right)\right]\right\} .
\end{aligned}
$$

L'élargissement Gaussien produit par l'imperfection du système optique est donné par

$$
F_{\mathrm{G}}(v)=\exp \left[-\left(\frac{v}{\Delta v_{\mathrm{G}}}\right)^{2}\right]
$$

où $\Delta v_{\mathrm{G}}$ exprime une largeur Gaussienne provenant principalement de la planéité de l'interféromètre. L'effet de l'appareillage est donc donné par

$$
F(v)=\int_{-\infty}^{\infty} F_{\mathrm{AP}}\left(v-v^{\prime}\right) F_{\mathrm{G}}\left(v^{\prime}\right) \mathrm{d} v^{\prime}
$$

5. - Profils spectraux observés. - Représentons l'intensité de spectre d'émission et celui d'absorption, leur élargissement par pression, leur largeur Doppler et leur fréquence centrale et leur déplacement de collision, par $I_{\mathrm{e}}(i), I_{\mathrm{a}}(i), \gamma_{\mathrm{fe}}(i), \gamma_{\mathrm{fa}}(i), \Delta v_{\mathrm{De}}(i), \Delta v_{\mathrm{Da}}(i), v_{\mathrm{Oe}}(i), v_{\mathrm{Oa}}(i), \Delta_{\mathrm{e}}(i)$ et $\Delta_{\mathrm{a}}(i)$, respectivement, le spectre d'émission, $f_{\mathrm{e}}(v)$ et le spectre d'absorption, $f_{\mathrm{a}}(v)$, sont donnés par (3), comme :

$$
\begin{aligned}
& f_{\mathrm{e}}(v)=\sum_{i} I_{\mathrm{e}}(i) \frac{\gamma_{\mathrm{fe}}(i)}{2 \pi \sqrt{\pi} \Delta v_{\mathrm{De}}(i)} \int_{-\infty}^{\infty} \frac{\exp \left[-\left(\frac{v-v^{\prime}}{\Delta v_{\mathrm{De}}(i)}\right)^{2}\right]}{\left[v^{\prime}-v_{\mathrm{oe}}(i)-\Delta_{\mathrm{e}}(i)\right]^{2}+\left[\frac{\gamma_{\mathrm{fe}}(i)}{2}\right]^{2}} \mathrm{~d} v^{\prime} \\
& f_{\mathrm{a}}(v)=\sum_{i} I_{\mathrm{a}}(i) \frac{\gamma_{\mathrm{fa}}(i)}{2 \pi \sqrt{\pi} \Delta v_{\mathrm{Da}}(i)} \int_{-\infty}^{\infty} \frac{\exp \left[-\left(\frac{v-v^{\prime}}{\Delta v_{\mathrm{Da}}(i)}\right)^{2}\right]}{\left[v^{\prime}-v_{\mathrm{oa}}(i)-\Delta_{\mathrm{a}}(i)\right]^{2}+\left[\frac{\gamma_{\mathrm{fa}}(i)}{2}\right]^{2}} \mathrm{~d} v^{\prime}
\end{aligned}
$$

où après substitutions de (6) et $\left(6^{\prime}\right)$ à $f_{\mathrm{e}}$ et $f_{\mathrm{a}}$ de l'éq. (4), on obtient :

$$
I_{\mathrm{a}}(v, h)=I_{\mathrm{a}}\left(f_{\mathrm{e}}=f_{\mathrm{e}}(v), f_{\mathrm{a}}=f_{\mathrm{a}}(v), h\right) .
$$

C'est le profil spectral de la lumière en dehors de l'axe.

Le profil spectral observé est donné, par (5) et (7), c'est-à-dire :

$$
I_{\mathrm{obs}}(v, h)=\int_{-\infty}^{\infty} F\left(v-v^{\prime}\right) I_{\mathrm{a}}\left(v^{\prime}, h\right) \mathrm{d} v^{\prime} .
$$

6. Structure hyperfine de la raie $D$ du $\mathbf{R b}$. - La structure hyperfine de la raie $D_{1}$ et $D_{2}$ du $R b$ est reprécertée par les figures 5 et 6 . En haut de chaque figure sont indiqués les niveaux d'énergie et les transitions dipolaires permises. En bas est figuré la distribution théorique d'intensité de chacune des composantes hyperfines sans élargissement. Le tableau I donne la position de chaque composante par rapport à la fréquence centrale. Des lettres capitales sont utilisées pour ${ }^{85} \mathrm{Rb}$ et des minuscules pour ${ }^{87} \mathrm{Rb}$. Les niveaux d'énergie et les positions des composantes sont donnés tous en unité de nombre d'onde (mk). Les chiffres sur les raies dans la figure de distribution d'intensité, expriment les intensités relatives. 

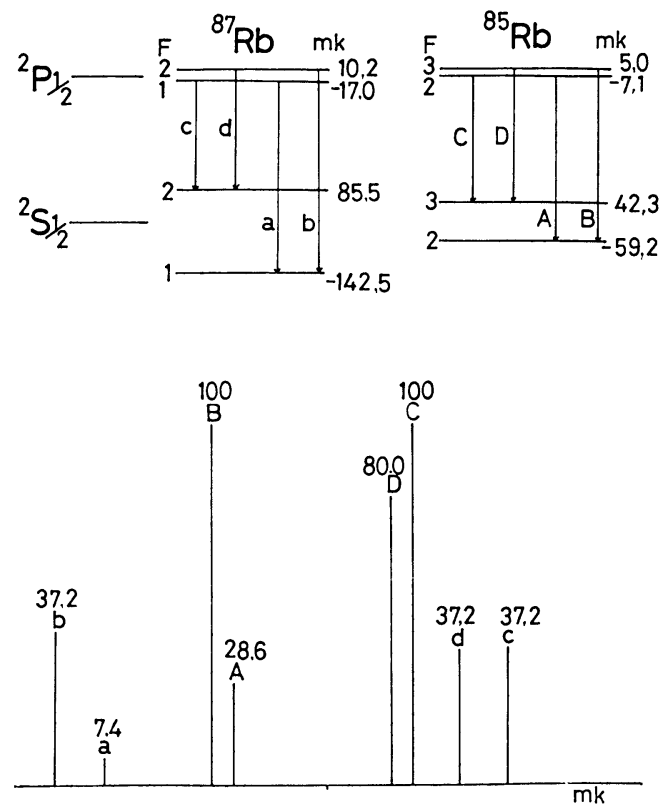

Fig. 5. - Structure hyperfine de la raie $D_{1}$ du $R b$ et distributions des intensités. Toutes les unités sont en mk. Les positions de chaque ligne sont montrées en fonction de la fréquence centrale de la raie $D_{1}$, sur le tableau $I$.

[Hyperfine structure of the Rb- $\mathrm{D}_{1}$ line and the intensity distributions. All the units are in mk. The positions of each of the lines are shown, as a function of the central frequency of $D_{1}$ line, in the table I.]
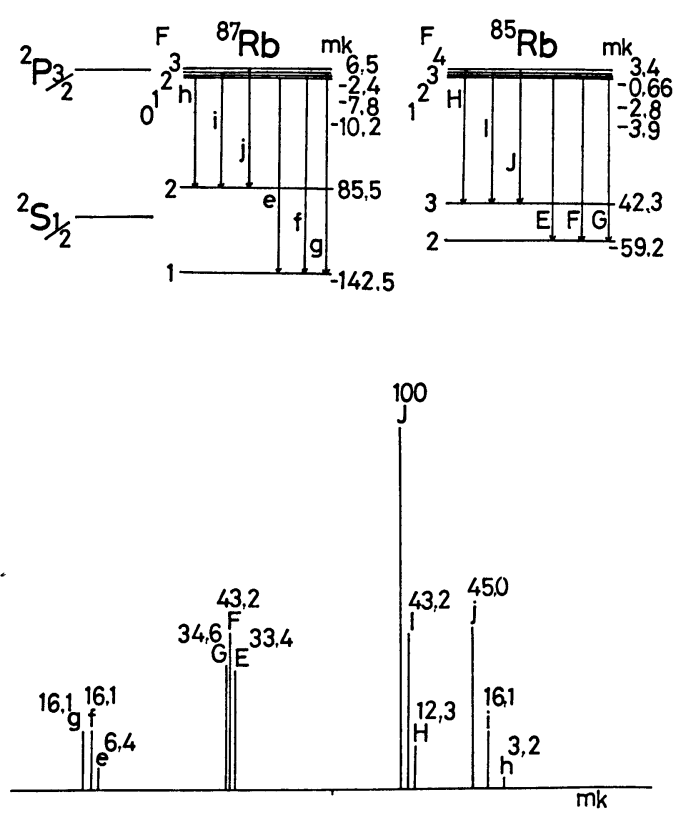

Fig. 6. - Structure hyperfine de la raie $\mathrm{D}_{2} \mathrm{du} \mathrm{Rb}$ et distributions des intensités. Toutes les unités sont en mk. Les positions de chaque ligne sont montrées en fonction de la fréquence centrale de la raie $D_{2}$, sur le tableau $I$.

[Hyperfine structure of the Rb- $\mathrm{D}_{2}$ line and the intensity distributions. All the units are in $\mathrm{mk}$. The positions of each of the lines are shown, as a function of the central frequency of $\mathrm{D}_{2}$ line, in the table I.]

\section{TABLEAU I}

Positions des composantes

[Positions of components]

\begin{tabular}{rrrrr} 
& & \multicolumn{1}{l}{$\mathrm{D}_{1}$} & & \multicolumn{1}{l}{$\mathrm{D}_{2}$} \\
& $\mathrm{~A}$ & $52,1 \mathrm{mk}$ & $\mathrm{E}$ & $55,3 \mathrm{mk}$ \\
& $\mathrm{B}$ & $64,2 \mathrm{mk}$ & $\mathrm{F}$ & $56,3 \mathrm{mk}$ \\
${ }^{85} \mathrm{Rb}$ & $\mathrm{C}$ & $-49,3 \mathrm{mk}$ & $\mathrm{G}$ & $58,5 \mathrm{mk}$ \\
& $\mathrm{D}$ & $-37,2 \mathrm{mk}$ & $\mathrm{H}$ & $-45,1 \mathrm{mk}$ \\
& & & $\mathrm{I}$ & $-43,0 \mathrm{mk}$ \\
& & & $\mathrm{J}$ & $-38,9 \mathrm{mk}$ \\
& & & & $132,3 \mathrm{mk}$ \\
& $\mathrm{a}$ & $125,5 \mathrm{mk}$ & $\mathrm{e}$ & $134,3 \mathrm{mk}$ \\
${ }^{87} \mathrm{Rb}$ & $\mathrm{b}$ & $152,7 \mathrm{mk}$ & $\mathrm{f}$ & $140,1 \mathrm{mk}$ \\
& $\mathrm{c}$ & $-102,8 \mathrm{mk}$ & $\mathrm{g}$ & $-93,3 \mathrm{mk}$ \\
& $\mathrm{d}$ & $-75,3 \mathrm{mk}$ & $\mathrm{h}$ & $-87,9 \mathrm{mk}$ \\
& & & $\mathrm{i}$ & $-79,0 \mathrm{mk}$
\end{tabular}

7. Résultats d'expérience. - Les profils des raies $D_{1}$ et $D_{2}$ du $R b$ émises par la lampe avec le gaz tampon Ar $267 \mathrm{~Pa}$, puissance continue 5,9 $\mathrm{W}$ et à la température mesurée de $387 \mathrm{~K}$, sont représentés sur la figure 7 . Dans cette figure, Centre correspond à $h \approx 0,0$, intermédiaire correspond à $h \approx 0,5$ et Bord à $h \approx 0,95$, c'est-à-dire au bord de la lampe.

Rb-Ar 267Pa, 387K
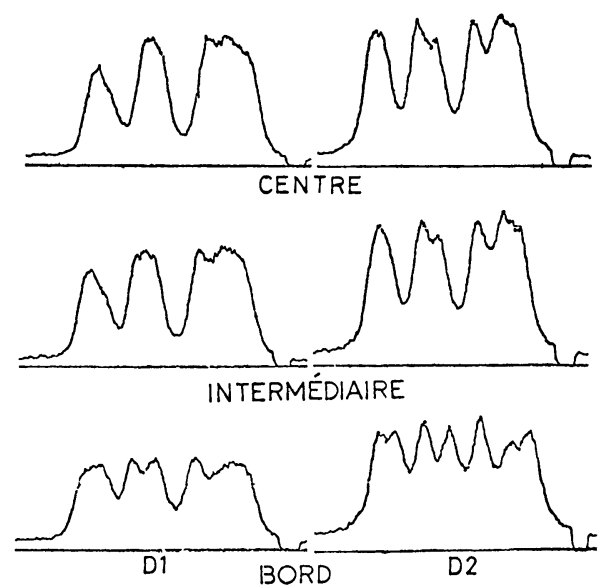

Fig. 7. - Profils spectraux mesurés, des raies $D_{1}$ et $D_{2}$ du $R b$ émises par la lampe Rb-Ar $267 \mathrm{~Pa}, 387 \mathrm{~K}$. Centre : cas où $h \approx 0,0$, Intermédiaire : cas où $h \approx 0,5$, Bord : cas où $h \approx 0,95$

[Measured spectral profiles of $D_{1}$ and $D_{2}$ lines of $R b$ emitted by the lamp Rb-Ar $267 \mathrm{~Pa}, 387 \mathrm{~K}$. Centre : for the case of $h \approx 0.0$, Intermediaire : for the case of $h \approx 0.5$, Bord : for the case of $h \approx 0.95$.]

L'absorption est d'autant plus forte qu'on s'éloigne du centre de la lampe. L'absorption de la raie $\mathrm{D}_{2}$ est plus forte que celle de $D_{1}$.

La figure 8 montre avec la même lampe les résultats de mesure des distributions radiales d'intensité de la 


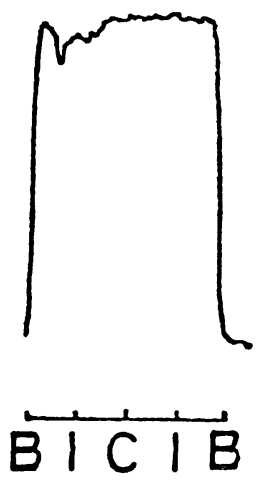

FIG. 8. - Distributions mesurées de l'intensité radiale de la raie $\mathrm{D}_{1} \mathrm{du} \mathrm{Rb}$. On montre les positions de la lampe observées ; $\mathrm{C}:$ centre $h \approx 0,0, \mathrm{I}:$ intermédiaire $h \approx 0,5, \mathrm{~B}:$ bord $h \approx 0,98$.

Pour Rb- $\mathrm{D}_{2}$, on observe à peu près la même distribution.

[Measured distributions of the radial intensity of the $D_{1}$ line of $\mathrm{Rb}$. The positions of the lamp observed are ; $\mathrm{C}$ : center $h \approx 0.0, \mathrm{I}:$ intermediate $h \approx 0.5$, B : edge $h \approx 0.98$. For Rb- $D_{2}$, almost the same distributions are observed.]

lumière, mais dans des conditions peut-être un peu différentes, car les figures 7 et 8 n'ont pas été mesurées en même temps. $\mathrm{C}$, I et B correspondent à $h \approx 0,0,0,5$ et 0,95 , respectivement. Comme la figure l'indique, la distribution d'intensité de la lumière n'a pas la forme d'une cloche, mais plutôt elle a tendance à être aplatie ou bien à avoir un creux au centre.

8. Détermination du profil des raies observées. On a utilisé ici la formule du trapèze qui donne une bonne précision du calcul numérique de l'intégration. L'ordinateur utilisé est MELCOM-COSMO 700.

Le tableau II indique les facteurs utilisés dans les calculs.

Pour calculer l'éq. (8), on suppose que la température soịt de $50^{\circ} \mathrm{C}$ lors du remplissage du gaz tampon dans la lampe, ce qui rend possible l'estimation du nombre d'atomes du gaz tampon dans la lampe et l'élargissement par collision avec le gaz tampon.

L'effet Doppler du second ordre, l'approximation dans l'aile et les collisions avec la paroi sont négligés. On suppose aussi que la fréquence centrale, la largeur naturelle, l'élargissement par collision avec le gaz tampon, la largeur Doppler et l'élargissement résonnant soient égaux pour le spectre d'émission et celui d'absorption mais que le déplacement soit différent pour l'un et pour l'autre. Ici, pour simplifier nous supposons que les déplacements de chaque composante hyperfine soient presque égaux et que l'effet Stark soit négligeable, ce qui est vérifié être une approximation suffisante par le résultat calculé ci-dessous.

Les profils obtenus par ce calcul sont représentés sur la figure 9, pour la lampe avec Rb-Ar $267 \mathrm{~Pa}$, puissance continue $5,9 \mathrm{~W}$, et la température mesurée $387 \mathrm{~K}$. Ces profils s'accordent assez bien avec ceux mesurés dans la figure 7 .

Les positions des composantes dans le tableau I et les facteurs dans le tableau II sont déterminés purement
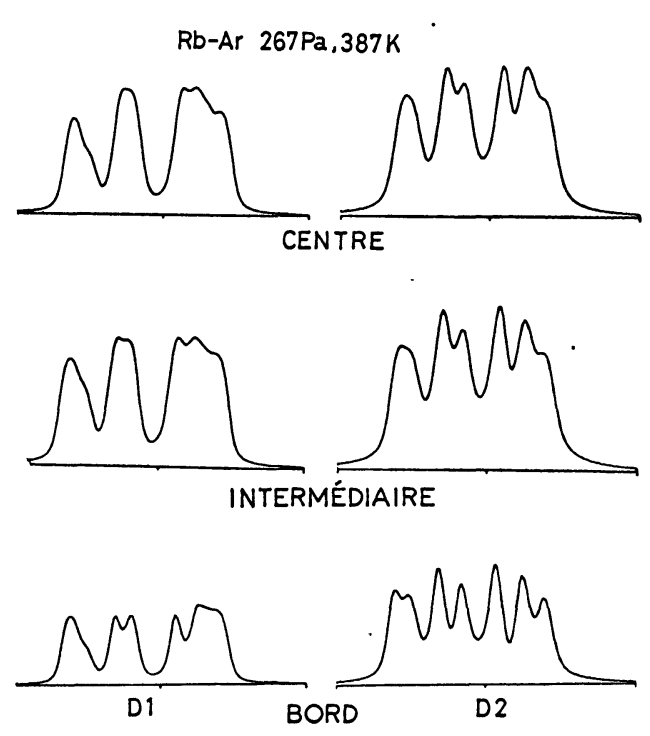

FIG. 9. - Profils spectraux théoriques des raies $D_{1}$ et $D_{2}$ du $R b$ émises par la lampe Rb-Ar $267 \mathrm{~Pa}, 387 \mathrm{~K}$. Centre : cas où $h=0,0$, Intermédiaire : cas où $h=0,5$, Bord : cas où $h=0,97$ $\left(\mathrm{D}_{1}\right), h=0,95\left(\mathrm{D}_{2}\right)$.

[Theoretical spectral profiles of $D_{1}$ and $D_{2}$ lines of $R b$ emitted by the lamp Rb-Ar $267 \mathrm{~Pa}, 387 \mathrm{~K}$. Centre : for the case of $h=0.0$, Intermediaire : for the case of $h=0.5$, Bord : for the case of $h=0.97\left(\mathrm{D}_{1}\right), h=0.95\left(\mathrm{D}_{2}\right)$.]

théoriquement. Dans ce calcul, les paramètres qu'on a variés pour obtenir des valeurs optima sont les suivants : le degré d'auto-absorption, $p$; les paramètres $a$ et $b$ qui concernent la distribution des atomes dans l'état excité et ceux dans l'état fondamental; $\Delta v_{\mathrm{G}}$, l'élargissement Gaussien, le déplacement entre le spectre d'émission et celui d'absorption; et la région $\mathrm{C}$ où des atomes dans l'état fondamental existent exclusivement.

\section{TABLEAU II}

\section{$R b-A r 267 P a$}

\begin{tabular}{lll} 
& \multicolumn{1}{c}{$\mathrm{D}_{1}$} & \multicolumn{1}{c}{$\mathrm{D}_{2}$} \\
$\nu_{0}$ & $1,2582 \times \overline{10}^{7} \mathrm{mk}$ & $1,2820 \times 10^{7} \mathrm{mk}$ \\
$\Gamma$ & $0,1869 \mathrm{mk}$ & $0,1940 \mathrm{mk}$ \\
$\gamma^{85 \mathrm{Rb})}$ & $0,1040 \times T^{0,3} \mathrm{mk}$ & $0,1040 \times T^{0,3} \mathrm{mk}$ \\
$\gamma^{87 \mathrm{Rb})}$ & $0,1033 \times T^{0,3} \mathrm{mk}$ & $0,1033 \times T^{0,3} \mathrm{mk}$ \\
$\Delta v_{\mathrm{D}}\left({ }^{85} \mathrm{Rb}\right)$ & $0,5853 \times T^{0,5} \mathrm{mk}$ & $0,5958 \times T^{0,5} \mathrm{mk}$ \\
$\Delta \nu_{\mathrm{D}}\left({ }^{87} \mathrm{Rb}\right)$ & $0,5758 \times T^{0,5} \mathrm{mk}$ & $0,5888 \times T^{0,5} \mathrm{mk}$ \\
$\gamma_{\mathrm{R}}$ & $2,23938 \times 10^{-14} N_{J_{0}} \mathrm{mk}$ & $4,39569 \times 10^{-14} N_{J_{0}} \mathrm{mk}$ \\
$\Delta v_{\mathrm{P}}$ & $0,7864 \mathrm{mk}$ & $0,8021 \mathrm{mk}$ \\
$R$ & 0,95 & 0,95
\end{tabular}

On suppose que le gaz tampon est rempli à $50^{\circ} \mathrm{C}=323 \mathrm{~K}$. $\mathrm{N}_{J}$ est donné par la formule de la pression de vapeur de $\mathrm{Rb}$. $M(85 \mathrm{Rb})=1,4195 \times 10^{-22} \mathrm{~g}, M(87 \mathrm{Rb})=1,4529 \times 10^{-22} \mathrm{~g}$.

[It is assumed that the buffer gas is filled at $50^{\circ} \mathrm{C}=323 \mathrm{~K}$. $N_{J_{0}}$ is given by the formula of the pressure of $\mathrm{Rb}$. $\left.M\left({ }^{85} \mathrm{Rb}\right)=1.4195 \times 10^{-22} \mathrm{~g}, M\left({ }^{87} \mathrm{Rb}\right)=1.4529 \times 10^{-22} \mathrm{~g}.\right]$ 
Les profils dans le cas où l'effet de l'appareillage n'est pas considéré, sont aussi représentés sur la figure 10 et calculés à l'aide de l'éq. (7). Par ailleurs, les profils qui n'ont pas l'effet d'auto-absorption sont représentés sur la figure 11 , qui sont calculés d'après l'éq. (3).
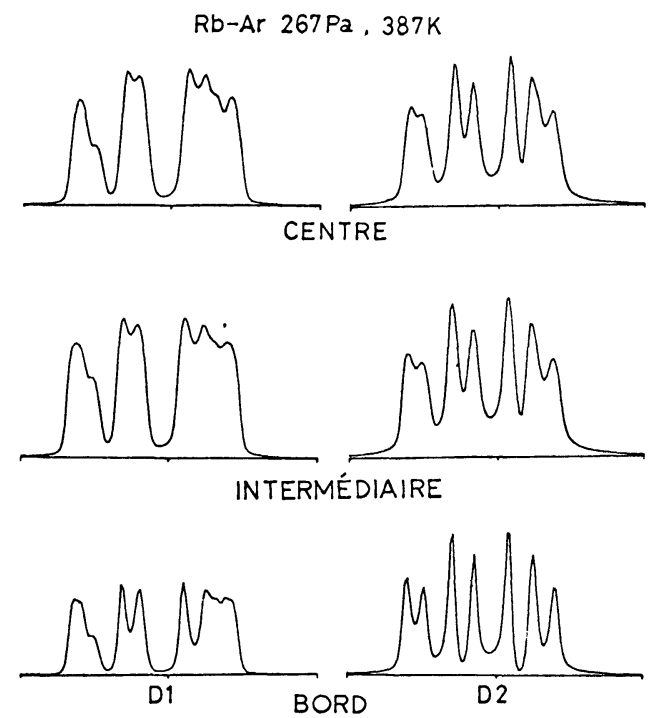

FIG. 10. - Profils spectraux quand on élimine l'effet d'appareil des profils de la figure 9.

[Spectral profiles when the effect of the instrument on the profiles in figure 9 is neglected.]

Cette détermination du profil donne $P=A N_{\mathrm{a}} \approx 1,3$, $a \approx 0,8$ et $b \approx 0,1$. Ces valeurs ne changent pas même si l'on utilise une autre formule [10] pour la pression de la vapeur de $R b$. L'élargissement Gaussien $\Delta v_{G}$ qui se produit par suite des imperfections du système optique est estimé environ $4 \mathrm{mk}$ pour la raie $D_{1}$ et environ $8 \mathrm{mk}$ pour la raie $\mathrm{D}_{2}$. Le déplacement entre le spectre d'émission et celui d'absorption est estimé être environ $1,2 \mathrm{mk}$.

Dans ce cas, on obtient la distribution radiale de l'intensité de la lumière (Fig. 12). La valeur de $C$ de la figure 3 est estimée être environ 0,98.

9. Discussion et conclusion. - Dans ces calculs, les paramètres s'obtiennent facilement par la méthode des moindres carrés quand il s'agit de fonctions polynomiales. Mais, les fonctions utilisées actuellement sont des convolutions de fonctions qui ne peuvent pas s'exprimer avec précision par quelques polynômes, ce qui explique qu'il n'y ait pas avantage à utiliser la méthode des moindres carrés pour obtenir des facteurs approximatifs.

De plus, le calcul est conduit en supposant que tous les atomes excités et dans l'état fondamental augmentent d'une façon quadratique du centre de la lampe vers la paroi, selon les résultats de van Tongeren [12].

Dans cette expérience, le champ électrique de haute fréquence est plus fort dans la région centrale de la
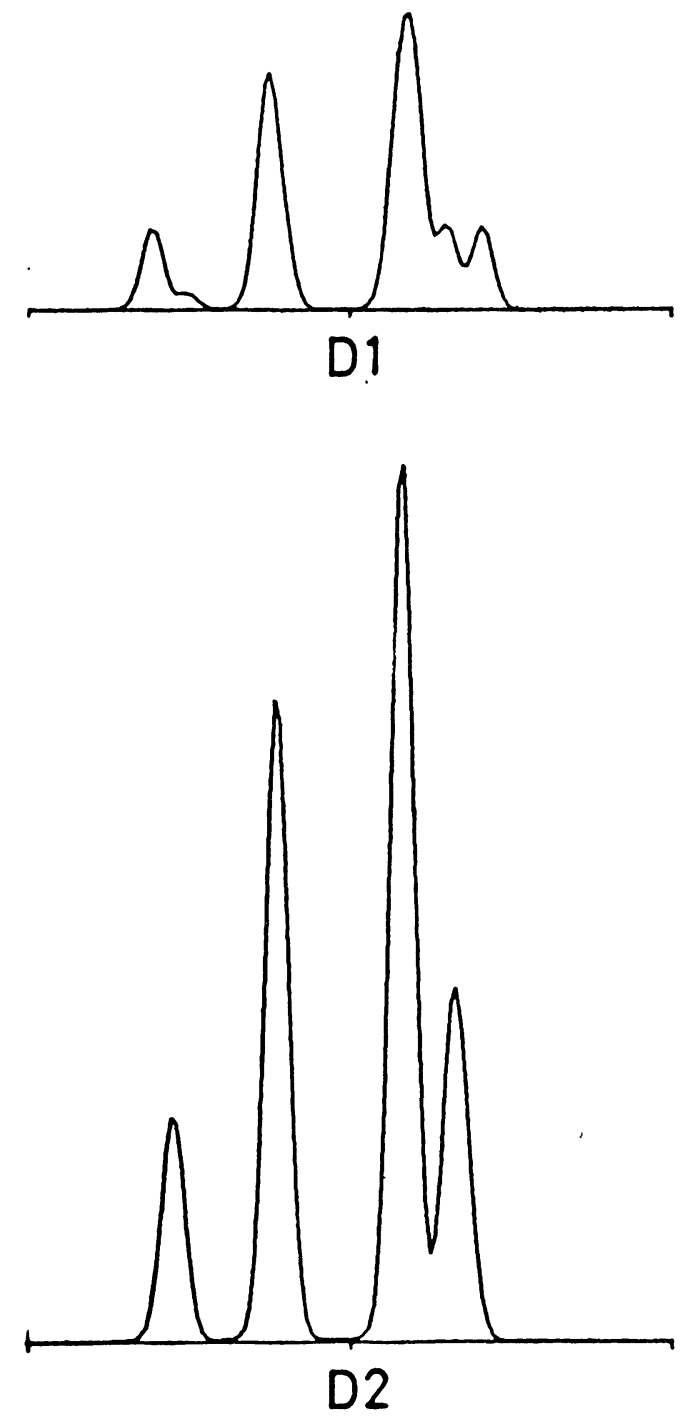

Fig. 11. - Profils spectraux, quand on élimine en outre l'effet d'auto-absorption des profils de la figure 10 .

[Spectral profiles, when the effet of self-absorption on the profiles in figure 10 is neglected.]

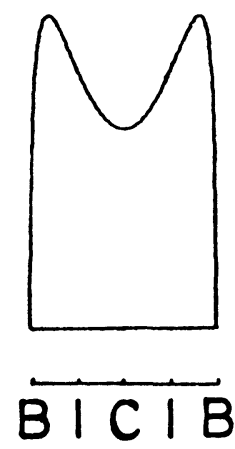

Fig. 12. - Distributions calculées de l'intensité radiale de la raie $\mathrm{D}$ du $\mathrm{Rb}$. La notation a le même sens que dans la figure 8 .

[Calculated distributions of the radial intensity distributions of the Rb-D line. The notation has the same meaning as in figure 8].

lampe où existent les atomes excités, ceux dans l'état fondamental et les ions. Les ions produisent un champ électrique qui déplace le niveau d'énergie de l'atome. 
Mais, l'accord entre le profil expérimental et celui théorique est suffisant, même si on néglige l'effet Stark.

On obtient ainsi le résultat suivant : les atomes dans l'état fondamental qui existent au centre sont en nombre inférieur de $20 \%$ environ à ceux de la paroi, tandis que les atomes excités n'existent presque jamais au centre, mais il peut y avoir quelques variations dans leurs valeurs. La distribution radiale de l'intensité de la lumière calculée est différente de celle mesurée de la figure 8 , mais, il semble que cette différence puisse provenir du fait que les figures 7 et 8 n'ont pas été mesurées simultanément.

L'effet Doppler du second ordre, l'élargissement et le déplacement par collision avec la paroi sont petits et négligés.

L'élargissement par collision et son déplacement avec le gaz tampon sont estimés être environ $0,62 \mathrm{mk}$ et $-0,22 \mathrm{mk}$, respectivement. La largeur naturelle est du même ordre que l'élargissement par collision avec le gaz tampon et son déplacement, ce qui explique que la largeur naturelle est un facteur non négligeable. L'élargissement résonnant a un grand effet sur l'élargissement de la raie $\mathrm{D}$ du $\mathrm{Rb}$ et augmente rapidement avec l'accroissement de température.

Pour la détermination du profil, il faut tenir compte de l'effet de l'appareillage et il est possible que dans certain cas l'effet du petit diaphragme et l'élargissement Gaussien dû à l'imperfection du système optique soient assez importants. L'élargissement Gaussien est plus petit que celui calculé directement par l'imperfection du miroir $\lambda / 100$, la région utilisée dans le miroir étant assez petite.

Dans l'éq. (4), on définit $A N_{\mathrm{a}}=p$, avec $p$ le degré d'auto-absorption [4]. Dans ce cas, $p$ est proportionnel au nombre d'atomes, dans l'état fondamental, qui se trouvent près de la paroi de la lampe. En général, si le nombre des atomes en fonction de la distance au centre de la lampe est exprimé par

$$
N_{\mathrm{a}}(r)=N_{\mathrm{a}} \cdot f(r)
$$

on peut supposer que $p \propto N_{\mathrm{a}}$. Donc, $p$ devient fonction de la température de la paroi et est proportionnel à $N_{J_{0}}$ donné par la formule de la pression de vapeur de $\mathrm{Rb}$. C'est pourquoi la valeur de $p$ peut être déduite de la mesure de la température de la paroi si la distribution des atomes dans l'état fondamental dans la lampe est connue.

Avec cette détermination du profil des raies, les paramètres modifiés dans le calcul sont $C, p, a, b, \Delta v_{\mathrm{G}}$ ainsi que le déplacement entre le spectre d'émission et celui d'absorption. Les autres facteurs sont obtenus purement théoriquement.

Le profil spectral des raies change de différentes manières avec la valeur de $p$, et le rapport des spectres d'absorption au centre, dans la région intermédiaire et au bord de la lampe change également avec la distribution des atomes qui est déterminée par les paramètres $a$ et $b$. D'autre part, $\Delta v_{\mathrm{G}}$ contribue à l'élargissement du spectre. Mais, quelles que soient les valeurs différentes qu'ils prennent, on ne peut pas obtenir l'asymétrie de composante $E, F$ et $G$ de la raie $\mathrm{D}_{2}$ (Fig. 6 et 7) et telle hauteur de l'intensité à gauche de la dépression produite par l'auto-absorption de composante $D, C, d$ et $c$ de la raie $\mathrm{D}_{1}$ (Fig. 5 et 7 ) non plus.

Cette difficulté peut être vaincue par l'introduction d'un déplacement entre $f_{\mathrm{e}}(v)$ et $f_{\mathrm{a}}(v)$ dans la lampe.

La valeur de ce déplacement est estimée être environ $1,2 \mathrm{mk}$ pour le cas traité actuellement et en tout cas certainement inférieure à 2 ou $3 \mathrm{mk}$ ainsi qu'on le vérifie par les calculs.

De nombreuses vérifications ont été faites pour établir que l'asymétrie observée sur $\mathrm{D}_{2}$ et la déformation de $\mathrm{D}_{1}$ ne sont pas dues aux erreurs expérimentales.

La distribution des atomes excités et ceux dans l'état fondamental n'est pas homogène dans la lampe. Chaque atome est dans une condition un peu différente l'une et l'autre. Donc, ce déplacement semble être observé.

Un effet important à considérer est l'existence d'atomes dans l'état fondamental dans une zone près de la paroi d'environ $100 \mu \mathrm{m}$.

Sans l'introduction d'une valeur finie pour $C$, les intensités des spectres au bord de la lampe se saturent et ses trois sommets s'arrangent horizontalement avec l'augmentation de la valeur de $p$. Seule l'introduction d'une valeur finie pour le paramètre $C$ enlève cette anomalie. La valeur de $C$ est déterminée par comparaison de l'effet d'auto-absorption entre les profils au centre, dans la région intermédiaire et au bord de la lampe.

Expérimentalement, on peut voir, un peu éloigné de la paroi, une zone qui émet une lumière plus forte (cf. Fig. 3). Les atomes excités effectuent des transitions de l'état fondamental à une faible distance de la paroi et il est possible que des ions et des électrons se combinent très près de la paroi. Par suite les atomes dans l'état fondamental s'accumuleront près de la paroi.

Ces résultats approximatifs seront un peu améliorés, si l'on tient compte que $f_{\mathrm{e}}(v)$ et $f_{\mathrm{a}}(v)$ varient à chaque point dans la lampe.

\section{Bibliographie}

[1] Margenau, H. et Watson, W. W., Rev. Mod. Phys. 8 (1936) 22.

[2] Ch'en, S. et Takeo, M., Rev. Mod. Phys. 29 (1957) 20.

[3] SobeL'MAN, I. I., Introduction to the theory of atomic spectra (Pergamon press) 1972.
[4] Tako, T., J. Phys. Soc. Japan 16 (1961) 2016.

[5] Cowan, R. D. et Dieke, G. H., Rev. Mod. Phys. 20 (1928) 638.

[6] Tako, T., Koga, Y. et Hirano, I., Japan. J. Appl. Phys. 14 (1975) 591. 
[7] Shimoda, K., Japan. J. Appl. Phys. 12 (1973) 1393.

[8] Smithells, C. J., ed., Metal Reference Book (Butterworths, London) 1962 p. 655.

[9] Tako, T., Koga, Y., HiRano, I. et OHI, M., Japan. J. Appl. Phys. 14 (1975) 1641.

[10] Hong, R. E. et Kramer, D. A., RCA Review 30 (1969) 285.
[11] Towns, C. H. et Schawlow, A. L., Microwave spectroscopy (McGraw-Hill) 1955 p. 374.

[12] Van Tongeren, H., J. Appl. Phys. 42 (1971) 3011.

[13] Van Tongeren, H., Phys. Lett., 37 A (1971) 317.

[14] Waszink, J. H. et Polman, J., J. Appl. Phys. 40 (1967) 2403.

[15] Gibis, H. M. et Hull, P. J., Phys. Rev. 153 (1967) 132.

[16] BIondi, M. A., Rev. Sci. Instrum. 27 (1956) 36. 